Chapman University

Chapman University Digital Commons

Psychology Faculty Articles and Research

Psychology

9-11-2018

\title{
Can Placental Corticotropin-Releasing Hormone Inform Timing of Antenatal Corticosteroid Administration?
}

Danielle A. Swales

University of Denver

Leah A. Grande

University of Denver

Deborah A. Wing

University of California, Irvine

Michelle Edelmann

University of Colorado

Laura M. Glynn

Chapman University,lglynn@chapman.edu

See next page for additional authors

Follow this and additional works at: https://digitalcommons.chapman.edu/psychology_articles

Part of the Maternal and Child Health Commons

\section{Recommended Citation}

Swales, D. A., Grande, L. A., Wing, D. A., et al. (2018). Can placental corticotropin-releasing hormone inform timing of antenatal corticosteroid administration? The Journal of Clinical Endocrinology \& Metabolism. doi: 10.1210/jc.2018-00956

This Article is brought to you for free and open access by the Psychology at Chapman University Digital Commons. It has been accepted for inclusion in Psychology Faculty Articles and Research by an authorized administrator of Chapman University Digital Commons. For more information, please contactlaughtin@chapman.edu. 


\section{Can Placental Corticotropin-Releasing Hormone Inform Timing of Antenatal Corticosteroid Administration?}

\section{Comments}

This is a pre-copy-editing, author-produced PDF of an article accepted for publication in The Journal of Clinical Endocrinology \& Metabolism following peer review. The definitive publisher-authenticated version

Swales, D. A., Grande, L. A., Wing, D. A., et al. (2018). Can placental corticotropin-releasing hormone inform timing of antenatal corticosteroid administration? The Journal of Clinical Endocrinology \& Metabolism. doi: $10.1210 /$ jc.2018-00956

is available online at DOI: $10.1210 /$ jc.2018-00956

\section{Copyright}

Endocrine Society

\section{Authors}

Danielle A. Swales, Leah A. Grande, Deborah A. Wing, Michelle Edelmann, Laura M. Glynn, Curt A.

Sandman, Roger Smith, Maria Bowman, and Elysia Poggi Davis 

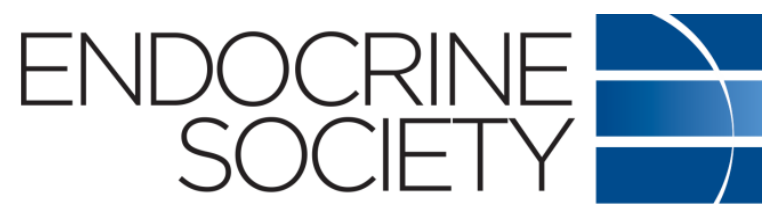

\title{
Can Placental Corticotropin-Releasing Hormone Inform Timing of Antenatal Corticosteroid Administration?
}

\author{
Danielle A. Swales, Leah A. Grande, Deborah A. Wing, Michelle Edelmann, Laura M. Glynn, \\ Curt Sandman, Roger Smith, Maria Bowman and Elysia Poggi Davis
}

\author{
The Journal of Clinical Endocrinology \& Metabolism \\ Endocrine Society
}

Submitted: April 30, 2018

Accepted: September 06, 2018

First Online: September 11, 2018

\begin{abstract}
Advance Articles are PDF versions of manuscripts that have been peer reviewed and accepted but not yet copyedited. The manuscripts are published online as soon as possible after acceptance and before the copyedited, typeset articles are published. They are posted "as is" (i.e., as submitted by the authors at the modification stage), and do not reflect editorial changes. No corrections/changes to the PDF manuscripts are accepted. Accordingly, there likely will be differences between the Advance Article manuscripts and the final, typeset articles. The manuscripts remain listed on the Advance Article page until the final, typeset articles are posted. At that point, the manuscripts are removed from the Advance Article page.
\end{abstract}

DISCLAIMER: These manuscripts are provided "as is" without warranty of any kind, either express or particular purpose, or non-infringement. Changes will be made to these manuscripts before publication. Review and/or use or reliance on these materials is at the discretion and risk of the reader/user. In no event shall the Endocrine Society be liable for damages of any kind arising references to, products or publications do not imply endorsement of that product or publication. 
Swales et al

Placental CRH Predicts Preterm Birth

\section{Can Placental Corticotropin-Releasing Hormone Inform Timing of Antenatal Corticosteroid Administration?}

Danielle A. Swales ${ }^{1 *}$ Leah A. Grande ${ }^{1 *}$ Deborah A. Wing ${ }^{2}$ Michelle Edelmann ${ }^{3}$ Laura M. Glynn ${ }^{4}$ Curt Sandman ${ }^{5}$ Roger Smith ${ }^{6}$ Maria Bowman ${ }^{6}$ and Elysia Poggi Davis ${ }^{1}$

${ }^{I}$ Department of Psychology, University of Denver, Denver, CO, USA; ${ }^{2}$ Obstetrics and Gynecology, University of California, Irvine, Orange, CA, USA $;^{3}$ University of Colorado, Anschutz Medical Campus, Aurora, CO, USA;

${ }^{4}$ Department of Psychology, Chapman University, Orange, CA, USA; ${ }^{5}$ Department of Psychiatry, University of

California, Irvine, Irvine, CA, USA;

${ }^{6}$ Mothers and Babies Research Centre, Hunter Medical Research Institute, Faculty of Health, University of Newcastle, NSW, Australia

Received 30 April 2018. Accepted 06 September 2018.

ORCiD numbers:

0000-0002-3187-9885

Swales

Danielle

0000-0001-6829-0495

Grande

Leah A.

*Danielle Swales and Leah Grande contributed equally to this work. This article has shared first authorship.

Context: Antenatal corticosteroids commonly are administered to pregnant women at risk of delivering between 23 and 34 gestational weeks, providing crucial benefits to fetal lung maturation and reducing risk of neonatal morbidity and mortality. Corticosteroids are maximally efficacious for lung maturation when administered within 2 to 7 days of delivery. Accurately identifying the timing of preterm delivery is thus critical to ensure that antenatal corticosteroids are administered within a week of delivery and to avoid unnecessary administration to women who will deliver at term. A plausible biomarker for predicting time of delivery is placental corticotropin-releasing hormone (pCRH). Objective: The current study assesses whether pCRH concentrations predict time to delivery, and specifically which women will deliver within a week of treatment. Design: pCRH concentrations were evaluated prior to administration of the corticosteroid betamethasone and timing of delivery was recorded. Participants: 121 women with singleton pregnancies who were prescribed betamethasone. Results: Elevated pCRH concentrations were associated with a shorter time from treatment to delivery. ROC curves revealed that pCRH may improve the precision of predicting preterm delivery. Conclusions: In the current sample, pCRH concentrations predicted the likelihood of delivering within one week of corticosteroid treatment. Current findings suggest that $\mathrm{pCRH}$ may be a diagnostic indicator of 
impending preterm delivery. Increasing the precision in predicting time to delivery could inform when to administer antenatal corticosteroids, thus maximizing benefits and reducing the likelihood of exposing fetuses who will be delivered at term.

Placental corticotropin-releasing hormone predicts impending delivery (i.e., delivery within a week) among pregnant women at risk for preterm birth.

\section{Introduction}

Preterm birth (i.e. delivery prior to 37 weeks gestation) is one of the leading causes of perinatal morbidity and mortality, as $12-13 \%$ of women deliver preterm each year within the United States. [1] Infants born preterm are at heightened risk for respiratory distress syndrome and other birth complications. The administration of corticosteroids to women at risk of preterm delivery has been the frontline of treatment to promote fetal lung maturation and ultimately decrease morbidity and mortality among preterm infants. [2] According to guidelines established by the American College of Obstetricians and Gynecologists (ACOG), corticosteroid treatment is efficacious for fetal lung maturation when administered antenatally to women who will deliver between $240 / 7$ and 33 6/7 weeks, [2,3] with recent data indicating a benefit through 36 6/7 weeks. [4] The benefits of antenatal corticosteroids are maximized when delivery occurs 2 to 7 days after the initial dose, with respiratory benefits dissipating after 7 days. [3,5] Corticosteroid treatment also is associated with reductions in neonatal mortality when delivery occurs within 24 hours of administration; [5] thus, treatment is recommended when women are at risk of delivering within 1 week. [2] There are clear benefits of treatment for infants born preterm, including reduced risk of respiratory distress syndrome, intraventricular hemorrhage, morbidity, and mortality. [3,6] However in addition to these benefits, antenatal corticosteroids may have long-term adverse developmental consequences, $[7,8]$ especially for infants who were at risk for preterm birth but proceeded to be delivered at term. Although negative effects of antenatal corticosteroids have not been observed in all studies, [2,8-10] administration of corticosteroids has been associated with fetal growth restriction, altered brain development, increased emotional reactivity, and persisting differences in stress physiology and hypothalamic-pituitary adrenal (HPA) axis dysregulation in human [7,8,22-27] and animal studies. [12-21]

Accurately identifying the risk for impending preterm delivery (i.e., delivery within a week) is critical for two reasons: first, to ensure that the timing of antenatal corticosteroid administration maximizes benefit to the fetus; and second, to avoid administering corticosteroids to women who will go on to deliver at term. As argued by Kamath-Rayne and colleagues, [28] improvement in prediction of the timing of preterm birth is needed so that administration of antenatal corticosteroids can occur within the ideal time frame of one week prior to delivery. We propose that placental corticotropin-releasing hormone ( $\mathrm{pCRH})$, a peptide postulated to lie on the pathway to preterm birth, could inform the timing of antenatal corticosteroid treatment. [29]

$\mathrm{CRH}$, a 41-amino-acid neuropeptide, is synthesized primarily in the paraventricular nucleus (PVN) of the hypothalamus and has a central role in regulating pituitary and adrenal function and the physiological response to stress. [30-32] During pregnancy, CRH also is synthesized and released from the placenta $(\mathrm{pCRH})$ and increases dramatically across the course of pregnancy, rising 25-fold through 31 weeks of gestation. [32-34] Placental CRH is identical in structure and activity to hypothalamic CRH. Concentrations of CRH in maternal circulation are almost exclusively of placental origin, because the quantity of hypothalamic CRH in maternal circulation is minute and rapidly degrades. [35,36] In the mother, pCRH binds to receptors in the pituitary and the adrenal glands, promoting the production of cortisol and 
dehydroepiandrosterone sulfate (DHEAS) and further release of pCRH from the placenta. Placental $\mathrm{CRH}$ also binds to receptors in the myometrium, stimulating contractile and relaxatory mechanisms. [30,35,37,38] In addition to effects on the mother, pCRH is released into the fetal compartment, binding to receptors in the pituitary and fetal zone of the adrenal, promoting the synthesis of cortisol and maturation of fetal lungs. Maturation of the fetal lungs is associated with increases in surfactant protein A, which may stimulate myometrial contraction. The increase of pCRH especially over the latter part of gestation, may therefore act upon various mechanisms to promote the onset of labor. Placental $\mathrm{CRH}$ has thus been implicated in the pathways underlying the timing of parturition. [29,39]

Prior work has established that pCRH predicts preterm birth in both low risk pregnancies, [29,35,39-40] as well as pregnancies at high risk for preterm delivery. [41] However, only two known studies have explored whether pCRH can predict delivery within days of assessment (e.g., 24 or 48 hours). [42,43] Korebrits and colleagues [42] report that elevated pCRH levels predict delivery within 24 hours; and Hill and colleagues [43] report that after 28 gestational weeks, elevated pCRH predicts delivery within 48 hours. Research, however, is needed to evaluate whether $\mathrm{pCRH}$ concentrations assessed prior to antenatal corticosteroid administration can inform the timing of treatment within the optimal window (i.e., whether delivery will occur within a week).

The current study evaluates whether pCRH concentrations prior to corticosteroid treatment can identify women at risk for impending preterm delivery (i.e., delivery within a week). We assess the specificity and sensitivity of pCRH in predicting timing of delivery among pregnant women prescribed betamethasone, the most commonly used antenatal corticosteroid. [44]

\section{Subjects and Methods}

\section{A. Study Overview}

We recruited women at risk for preterm delivery who were prescribed betamethasone between 23 0/7 and 34 0/7 gestational weeks. Placental CRH levels were evaluated prior to betamethasone administration, and date of delivery was determined via medical record abstraction. Study protocols were reviewed and received approval from the Institutional Review Board. Written and informed consent was obtained from the mother before study enrollment.

\section{B. Participants}

Participants included 121 women at risk for preterm delivery with singleton pregnancies who were prescribed betamethasone, from the University of California, Irvine Medical Center (UCIMC). Exclusion criteria included: a known HPA axis or endocrine disorder, self-reported maternal substance use, prior corticosteroid exposure during this pregnancy, in vitro fertilization, and major fetal or chromosomal anomalies. During the recruitment period (from 2011 to 2014), $43.8 \%$ of those women treated with betamethasone failed to meet eligibility criteria and were not recruited. Among the eligible women who were approached for participation $18.8 \%$ declined due to various reasons, the most common of which were: study burden, feeling overwhelmed and pregnancy-related worries.

The decision to administer betamethasone rested entirely on the clinical judgment of the attending obstetrician and was made independent of study participation. As per standardized hospital protocol, the attending obstetrician evaluated preterm labor at less than 34 weeks, administered temporizing measures such as parenteral magnesium sulfate or oral nifedipine, and determined whether betamethasone treatment was needed. If deemed necessary, betamethasone was administered in 2 doses (12 mg intramuscular, 24 hours apart). Consistent with standard of 
care, the first dose of betamethasone was given to participants between 23 and 34 weeks' gestation $(\mathrm{M}=28.4, \mathrm{SD}=0.3$ weeks $)$. On average, delivery occurred 42 days after the first dose of betamethasone administration ( $\mathrm{SD}=31.0$ days, Range [0-104]).

\section{Placental CRH assessment}

Maternal blood samples were collected for placental CRH, on average, 36 minutes prior to betamethasone treatment (Range [1min to $4 \mathrm{hrs} 16 \mathrm{~min}]$ ). $50 \mu \mathrm{L}$ of protease inhibitor, Aprotinin (Thermo Fisher Scientific) were added to the plasma tubes and refrigerated. After 30 minutes, tubes were centrifuged, processed, and frozen at $-70^{\circ} \mathrm{C}$. Plasma pCRH assays were completed by Dr. Roger Smith's lab at the University of Newcastle, Australia. Placental CRH concentrations (pg/ml) were analyzed by radioimmunoassay (RIA) as previously described. [29] Extraction recovery was $82.5 \%$. No correction of the data for extraction recoveries was made. Inter- and intra-assay coefficients of variance were $9.8 \%$ and $7.6 \%$ respectively. Data reduction for the RIA assay was with a computer assisted logistics program.

Analyses were performed both with raw pCRH data and with log transformed data, due to skewness. Statistical significance was maintained whether untransformed or transformed data were used. Raw data are thus presented here in nanomoles per liter (nmol/L) to improve interpretability.

\section{Sociodemographic and medical characteristics}

Sociodemographic characteristics including marital and cohabitation status, education, and household income were determined at the time of study entry by maternal interview. Family income was assessed based on an income-to-needs ratio, calculated by dividing total household income by the poverty threshold at the time of assessment, specified by the U.S. Census Bureau.

Medical records were reviewed to determine obstetric and neonatal medical characteristics. Body mass index (BMI) was calculated based on maternal height and weight in the third trimester, or within one month of delivery if the mother delivered prior to the third trimester $\left(\mathrm{BMI}=(\text { height } / \text { weight })^{2}\right)$. Primary reason for betamethasone treatment was grouped into three categories: inflammation, placental issues, and utero-placental insufficiency. The inflammation group included women with preterm labor, short cervix, preterm premature rupture of membranes (PPROM), chorioamnionitis, or a positive fetal fibronectin test. The placental issues group included women with placenta previa, abruption, accreta, or oligohydramnios. The uteroplacental insufficiency group included women with pre-eclampsia, chronic hypertension, gestational hypertension, pregnancy-induced hypertension, intrauterine growth restriction, or abnormal end diastolic flow results from umbilical artery Doppler velocimetry.

\section{E. Data Analyses}

As expected, pCRH concentrations were positively correlated with gestational age (GA) at time of assessment $(\mathrm{r}=0.65, P<0.001)$. [38] Thus, all analyses with pCRH covaried with GA at time of assessment. The following variables were evaluated as potential covariates and were included in the models if they were significantly associated $(P<0.10)$ with both predictor $(\mathrm{pCRH})$ and outcome (time from betamethasone administration to delivery) after adjusting for GA at assessment: maternal age, cohabitation, ethnicity, education level, income-to-needs ratio, living below the federal poverty line, pregnancy history, and late pregnancy BMI. None of these additional variables met criterion for inclusion as a covariate.

We first used partial correlations in IBM SPSS Statistics for Windows, version 23 (IBM Corp., Armonk, N.Y., USA) to evaluate whether pCRH concentrations were associated with time from treatment to delivery and gestational age at birth (GAB). Linear regression was employed 
using SPSS as well to evaluate whether the reason for betamethasone administration accounted for study findings (i.e. reason for betamethasone was included as a covariate in a linear regression model with pCRH concentrations as the predictor and time from treatment to delivery as the outcome variable) or moderated the association between pCRH and time from treatment to delivery (i.e. an interaction term was created between reason for betamethasone treatment and pCRH concentrations, which was then added to the linear regression model). Next, Cox proportional hazard models were fitted to investigate whether pCRH concentrations predicted risk for delivering over time. Cox proportional hazard models were performed using SAS software for Unix (SAS Institute Inc., Cary, NC, USA). Guidelines from ACOG during the study period recommend that corticosteroids be administered to pregnant women between $240 / 7$ and 33 6/7 weeks of gestation, who are at risk of delivering within a week. [2] Thus, the sample was divided into two groups: women who delivered within a week of treatment $(n=25)$ and those who delivered after a week $(n=96)$. Computed receiver operating characteristic (ROC) curves assessed the sensitivity and specificity of pCRH concentrations in predicting whether or not women would deliver within a week. These models were performed using SAS software. The Youden index (sensitivity + [specificity-1]) was computed to obtain a cutoff score that optimizes differentiating ability when equal weight is given to sensitivity and specificity. Since optimizing sensitivity is a priority for clinical practice, we also identified an alternative cutoff that weighed sensitivity over specificity.

\section{Results}

\section{A. Sample Characteristics}

Demographic characteristics of study participants are summarized in Table 1 . Women were, on average, 30 years of age at recruitment $(S D=6.6$; Range [18-46 years]), primarily Latina $(63.6 \%)$, and the majority were cohabitating with a partner $(81 \%)$. The range of the income-toneeds ratio was 0 to 33.19. Approximately 1 in 3 participants were living below the federal poverty line (income-to-needs ratio below 1.00).

\section{B. Time from Treatment to Delivery}

All 121 women were prescribed betamethasone at the independent discretion of the attending physician and in adherence with current ACOG guidelines. Risk status was thus determined entirely by the physician and not in relation to the current study protocol. Of these women, 25 $(20.7 \%)$ delivered within a week of treatment. Additionally, $69(57 \%)$ women went on to deliver preterm and 52 (43\%) delivered at term (see Table 2).

\section{Corticotropin-Releasing Hormone}

Women with elevated pCRH concentrations were more likely to delivery earlier $(r=-0.35, P<$ $0.001)$ and were more likely to have a shorter time between treatment and delivery $(r=-0.35, P$ $<0.001$ ); see supplemental figures S1A and S1B in the online data repository for correlation plots. [45] Further, pCRH concentrations were higher among women who subsequently delivered preterm $(\mathrm{M}=86.87, \mathrm{SD}=127.34)$ as compared to women who delivered full term (M $=47.28, \mathrm{SD}=43.90 ; \mathrm{F}=9.51, P=0.003)$. Placental $\mathrm{CRH}$ concentrations were also higher among women who delivered within the week $(\mathrm{M}=170.89 \mathrm{pmol} / \mathrm{L}, \mathrm{SD}=178.45)$ in as compared to those who $\operatorname{did} \operatorname{not}(\mathrm{M}=44.86 \mathrm{pmol} / \mathrm{L}, \mathrm{SD}=46.70 ; \mathrm{F}=42.13, P<0.001)$. For the subset of women who remained pregnant at least a week after treatment, pCRH showed the expected increase with advancing gestation $(\mathrm{t}(89)=-6.194, P<0.001)$. However, pCRH levels were highly correlated across this one-week period $(r=0.971, P<0.001)$. 
Reason for betamethasone administration (i.e., inflammation, placental issues, uteroplacental insufficiency) did not account for study findings, as pCRH was still a significant predictor of time from treatment to delivery after covarying reason for betamethasone administration $(t(117)=-1.32, \mathrm{P}=0.189)$. Further, reason for betamethasone administration did not moderate the association between $\mathrm{pCRH}$ concentrations and time from treatment to delivery $(\beta=-0.335, P>0.250)$.

Cox hazard modeling revealed that higher concentrations of $\mathrm{pCRH}$ were associated with earlier delivery $(\beta=0.0058, \mathrm{SE}=0.001, P<0.0001,95 \% \mathrm{CI}=0.0036-0.0079)$. Data were analyzed continuously, however for illustrative purposes are presented as the top and bottom quartiles of pCRH concentrations in Figure 1. For every increase of 10pmol/L of pCRH, the risk of delivery (i.e., delivery within a week) was $5.8 \%$ higher.

ROC curves reveal that $\mathrm{pCRH}$ concentrations may improve the precision of predicting preterm delivery, among a sample of women who received betamethasone under current standard of care (area under the curve $(\mathrm{AUC})=0.79,95 \% \mathrm{CI}=0.68-0.90, P=0.001$; Figure $2 \mathrm{~A}$ ). The ROC model was used to determine optimal pCRH level cutoffs for these data, to predict which women were likely to deliver within a week. When specificity was weighted equally with sensitivity (maximizing the Youden index), the optimal cutoff score for pCRH for this sample with this assay was $72.8 \mathrm{pmol} / \mathrm{L}$ (Youden index $=0.52$ ). At this cutoff, $72 \%$ of women in this sample who delivered within one week would have been correctly identified (sensitivity), whereas $20 \%$ of women who delivered after one week would have been falsely identified (1specificity). See Figure S2A in the online data repository for Kaplan-Meier plots showing the probability of remaining pregnant for women with pCRH concentrations above and below the Youden index cut-off.

However, providing betamethasone to women who will deliver within a week (sensitivity) is a priority for clinical care and available tools to predict preterm birth are limited. Therefore, to prioritize sensitivity while maintaining a reasonable degree of specificity, we identified an alternative cutoff value of $15.5 \mathrm{pmol} / \mathrm{L}$ for this sample. At this cutoff, $96 \%$ of women who delivered within one week would have been correctly identified as delivering within this window (sensitivity), whereas $69 \%$ of women who delivered after one week would have been falsely identified (1-specificity). Additionally, if a pCRH cutoff of $15.5 \mathrm{pmol} / \mathrm{L}$ were applied to the current study sample, 30 women who delivered more than a week after betamethasone administration would have avoided unnecessary exposure, 14 of whom delivered at term gestation. See Figure S2B in the online data repository for Kaplan-Meier plots showing the probability of remaining pregnant for women with pCRH concentrations above and below the alternative cut-off.

\section{Discussion}

Our study findings suggest that pCRH concentrations prior to betamethasone treatment predict impending preterm delivery. Specifically, among women admitted to the hospital for threatened preterm labor and given corticosteroids, elevated pCRH concentrations prior to treatment improved discrimination between women who would deliver within a week and those who would not. The critical importance of this issue is highlighted by the fact that $43 \%$ of women in our sample delivered at term gestation. Because of challenges predicting timing of delivery, there has been a call for new tools and biomarkers to identify optimal timing of antenatal corticosteroid administration. [28] The present findings indicate that pCRH could be an 
additional tool that contributes to the determination of risk for impending preterm delivery and thus, informs timing of corticosteroid administration.

Decades of research have established that antenatal corticosteroids are a lifesaving treatment for preterm infants. [2,5,46] Antenatal corticosteroids provide multiple benefits to fetal health and development by stimulating fetal maturation and reducing risk of neonatal morbidity and mortality. [2,5,46] The benefits of antenatal corticosteroids are greatest when the infant is delivered within 7 days following treatment. [3,5] However, exposure to corticosteroids may be associated with elevated risk for neurodevelopmental delays, [7,8,22-27] especially in cases where the infant was at risk for preterm delivery but delivered at term.

Because of the challenges of predicting length of gestation and preterm birth, [28] further investigation into biological measures predicting the onset of parturition is needed. Pretreatment levels of pCRH show promise in addressing this clinical need, because they predict preterm birth and play an integral role in the causal pathways of parturition. [39] Specifically, our findings suggest that pCRH may improve our ability to identify women who will go on to deliver within a week, so that infants born preterm receive maximum benefit from corticosteroid treatment and infants born term avoid unnecessary exposure. In the current sample, a cutoff of $15.5 \mathrm{pmol} / \mathrm{L}$ correctly identified $96 \%$ of women who delivered within a week, with a rate of misclassification of $69 \%$. Notably, based on current standard of care, all women within this sample were administered corticosteroids, although $79 \%$ did not deliver within a week of treatment. Using the pCRH cutoff of $15.5 \mathrm{pmol} / \mathrm{L}, 31 \%$ of currently treated women would have been appropriately identified as not benefiting from treatment at this time. Findings highlight the potential clinical utility of pCRH in identifying if and when offspring may benefit from treatment.

The possible utility of pCRH as a clinical indicator of impending preterm delivery is supported by evidence that pCRH is part of the causal pathway controlling length of gestation. [29] Placental CRH concentrations increase dramatically over the course of pregnancy. [39] Placental CRH binds to receptors promoting myometrial relaxation; however, as the pregnancy progresses, these receptors become less effective and ultimately activate contractile pathways. [30] Because of the robust association between pCRH and timing of delivery, pCRH has been characterized as part of a "placental clock" controlling the onset of parturition. [31,39]

To our knowledge, only two studies have used pCRH to prospectively predict delivery within a set time frame (e.g., 24 and 48 hours). [42,43] These studies suggest that pCRH after 28 weeks' gestation predicts delivery within 24 to 48 hours. [42,43] The present results extend these findings by suggesting that pCRH is a useful biomarker that can aid the decision of whether to administer corticosteroids by identifying women who will deliver in less than a week.

Although findings suggest that pCRH could potentially serve as a diagnostic indicator of impending preterm delivery, several limitations should be addressed. First, validation studies with larger samples sizes and across varying populations (e.g. in low risk pregnancies) are needed in order to replicate the findings of this single-site investigation and to evaluate clinical utility. Second, in our sample, the indications for corticosteroid administration were heterogeneous and we did not find that the reason for treatment moderated the relation between pCRH and time from treatment to delivery. A strength of this approach is that it increases generalizability of our findings to a more medically diverse population of women; however, future studies should carefully evaluate these and other clinical risk factors in study design and interpretation of findings. Third, the current study recruited women with singleton pregnancies. The relevance to multifetal pregnancies thus remains unknown. Fourth, it is also unclear if pCRH could be combined with other clinical indicators of preterm birth, including 
biomarkers such as placental alpha microglobulin-1 (PAMG-1), fetal fibronectin, and transvaginal cervical length measurements, to optimize the ability to predict gestational length. Exploration of the possible advantages of utilizing multiple indicators of risk for preterm birth could further improve accuracy in predicting preterm delivery.

The findings of this study could have important implications for obstetric practice. If these results are replicated work could be done to develop a cost-effective and clinically feasible pCRH screen and evaluate its clinical utility in obstetric practice. Enzyme-linked immunosorbent assays (ELISAs) have been developed for assessing CRH and future work could explore the feasibility of this method as a faster and more cost-effective approach. [47] Placental CRH taps in to the causal pathways of delivery, which makes it a promising biomarker for predicting impending preterm delivery. More effective clinical tools to predict the timing of parturition could guide when to administer corticosteroids and increase the likelihood of providing treatment within the optimal time frame of one week prior to preterm delivery. This would maximize benefits to preterm neonates, improving lung maturation and reducing rates of mortality and respiratory distress syndrome. Further, more precise prediction of timing of delivery would reduce the likelihood of administering antenatal corticosteroids to women who will go on to deliver at term, and thus whose offspring will not benefit from treatment. A current clinical goal is to optimize the sensitivity and specificity of clinical indicators of impending preterm delivery. $[2,28]$ pCRH may be a promising biomarker to inform the timing of antenatal corticosteroid treatment, which would maximize the likelihood of delivering treatment within the optimal window.

\section{Acknowledgments}

This research was supported by the National Institutes of Health [RO1 HD065823; P50MH 096889]. The assistance of Megan Faulkner, Kendra Leak and Natalie Hernandez for their participation in data collection is gratefully acknowledged. The authors thank the families who participated in this project.

This research was supported by the National Institutes of Health [RO1 HD065823; P50MH 096889].

National Institutes of Health http://dx.doi.org/10.13039/100000002, RO1 HD065823, Elysia Poggi Davis; National Institutes of Health http://dx.doi.org/10.13039/100000002, P50MH 096889, Elysia Poggi Davis

Correspondence: Danielle A. Swales, Department of Psychology, University of Denver, Frontier Hall 2155 S. Race St., Denver CO 80206, USA, Tel +1 303-871-3797, Fax +1 303-871-4747, Email: daswales@gmail.com

Disclosure.

The authors report no conflicts of interest in this work and have nothing to disclose.

\section{References}

1. Goldenberg, RL, Culhane, JF, Iams, JD, Romero, R. Epidemiology and causes of preterm birth. The lancet, 2008;371(9606), 75-84.

2. American College of Obstetricians and Gynecologists. Antenatal corticosteroid therapy for fetal maturation: Committee Opinion No. 677. Obstet Gynecol. 2016;128; 187-94.

3. Crowley PA. Antenatal corticosteroid therapy: a meta-analysis of the randomized trials, 1972 to 1994. Am J Obstet Gynecol. 1995;173(1):322-335. 
4. Gyamfi-Bannerman C, Thom EA, Blackwell SC, et al. Antenatal betamethasone for women at risk for late preterm delivery. N Engl J Med. 2016;374(14):1311-1320.

5. Roberts D, Dalziel S. Antenatal corticosteroids for accelerating fetal lung maturation for women at risk of preterm birth. Cochrane Database Syst Rev. 2006;(3):CD004454.

6. Baud O. Antenatal corticosteroid therapy: benefits and risks. Acta Padiatrica. 2004;93:610.

7. Alexander N, Rosenlöcher F, Stalder T, et al. Impact of antenatal synthetic glucocorticoid exposure on endocrine stress reactivity in term-born children. J Clin Endocrinol Metab. 2012;97(10):3538-3544.

8. Davis EP, Sandman CA, Buss C, Wing DA, Head K. Fetal glucocorticoid exposure is associated with preadolescent brain development. Biol Psychiatry. 2013;74(9):647-655.

9. Lee BH, Stoll BJ, McDonald SA, Higgins RD. Neurodevelopmental outcomes of extremely low birth weight infants exposed prenatally to dexamethasone versus betamethasone. Pediatrics. 2008;121(2):289-296.

10. Schmand B, Neuvel J, Smolders-de Haas H, Hoeks J, Treffers PE, Koppe JG.

Psychological development of children who were treated antenatally with corticosteroids to prevent respiratory distress syndrome. Pediatrics. 1990;86(1):58-64.

11. Smolders-de Haas H, Neuvel J, Schmand B, Treffers PE, Koppe JG, Hoeks J. Physical development and medical history of children who were treated antenatally with corticosteroids to prevent respiratory distress syndrome: a 10- to 12-year follow-up. Pediatrics. 1990;86(1):65-70. 12. Jobe AH, Wada N, Berry LM, Ikegami M, Ervin MG. Single and repetitive maternal glucocorticoid exposures reduce fetal growth in sheep. Am J Obstet Gynecol. 1998;178(5):880885.

13. Johnson JW, Mitzner W, London WT, Palmer AE, Scott R. Betamethasone and the rhesus fetus: multisystemic effects. Am J Obstet Gynecol. 1979;133(6):677-684.

14. Scheepens Arjan, Waarenburg Marjo, Hove Daniël, Blanco Carlos E. A single course of prenatal betamethasone in the rat alters postnatal brain cell proliferation but not apoptosis. $J$ Physiol. 2004;552(1):163-175.

15. Slotkin TA, Zhang J, McCook EC, Seidler FJ. Glucocorticoid administration alters nuclear transcription factors in fetal rat brain: implications for the use of antenatal steroids. Brain Res Dev Brain Res. 1998;111(1):11-24.

16. Ferguson SA, Holson RR. Neonatal dexamethasone on day 7 causes mild hyperactivity and cerebellar stunting. Neurotoxicol Teratol. 1999;21(1):71-76.

17. Muneoka K, Mikuni M, Ogawa T, et al. Prenatal dexamethasone exposure alters brain monoamine metabolism and adrenocortical response in rat offspring. Am J Physiol-Regul Integr Comp Physiol. 1997;273(5):R1669-R1675.

18. Sloboda DM, Moss TJ, Gurrin LC, Newnham JP, Challis. The effect of prenatal betamethasone administration on postnatal ovine hypothalamic-pituitary-adrenal function. $J$ Endocrinol. 2002;172(1):71-81.

19. Benesová O, Pavlík A. Perinatal treatment with glucocorticoids and the risk of maldevelopment of the brain. Neuropharmacology. 1989;28(1):89-97.

20. Nagano M, Ozawa H, Suzuki H. Prenatal dexamethasone exposure affects anxiety-like behaviour and neuroendocrine systems in an age-dependent manner. Neurosci Res. 2008;60(4):364-371. 
21. Oliveira M, Bessa JM, Mesquita A, et al. Induction of a hyperanxious state by antenatal dexamethasone: A case for less detrimental natural corticosteroids. Biol Psychiatry. 2006;59(9):844-852.

22. Bloom SL, Sheffield JS, McIntire DD, Leveno KJ. Antenatal dexamethasone and decreased birth weight. Obstet Gynecol. 2001;97(4):485-490.

23. Davis EP, Waffarn F, Uy C, Hobel CJ, Glynn LM, Sandman CA. Effect of prenatal glucocorticoid treatment on size at birth among infants born at term gestation. $J$ Perinatol Off $J$ Calif Perinat Assoc. 2009;29(11):731-737.

24. Thorp JA, Jones PG, Knox E, Clark RH. Does antenatal corticosteroid therapy affect birth weight and head circumference? Obstet Gynecol. 2002;99(1):101-108.

25. Davis EP, Townsend EL, Gunnar MR, et al. Effects of prenatal betamethasone exposure on regulation of stress physiology in healthy premature infants. Psychoneuroendocrinology. 2004;29(8):1028-1036.

26. Davis EP, Townsend EL, Gunnar MR, et al. Antenatal betamethasone treatment has a persisting influence on infant HPA axis regulation. J Perinatol Off J Calif Perinat Assoc. 2006;26(3):147-153.

27. Edelmann MN, Sandman CA, Glynn LM, Wing DA, Davis EP. Antenatal glucocorticoid treatment is associated with diurnal cortisol regulation in term-born children.

Psychoneuroendocrinology. 2016;72:106-112.

28. Kamath-Rayne BD, Rozance PJ, Goldenberg RL, Jobe AH. Antenatal corticosteroids beyond 34 weeks gestation: What do we do now? Am J Obstet Gynecol. 2016;215(4):423-430. 29. Smith R, Smith JI, Shen X, et al. Patterns of plasma corticotropin-releasing hormone, progesterone, estradiol, and estriol change and the onset of human labor. J Clin Endocrinol Metab. 2009;94(6):2066-2074.

30. Smith R, Nicholson RC. Corticotrophin releasing hormone and the timing of birth. Front Biosci J Virtual Libr. 2007;12:912-918.

31. Smith R. Parturition. N Engl J Med. 2007;356(3):271-283.

32. Frim DM, Emanuel RL, Robinson BG, Smas CM, Adler GK, Majzoub JA.

Characterization and gestational regulation of corticotropin-releasing hormone messenger RNA in human placenta. J Clin Invest. 1988;82(1):287-292.

33. Chrousos GP. Regulation and dysregulation of the hypothalamic-pituitary-adrenal axis. The corticotropin-releasing hormone perspective. Endocrinol Metab Clin North Am. 1992;21(4):833-858.

34. Vale W, Spiess J, Rivier C, Rivier J. Characterization of a 41-residue ovine hypothalamic peptide that stimulates secretion of corticotropin and $\beta$-endorphin. Science.

1981;213(4514):1394-1397.

35. Sandman CA, Glynn L, Schetter CD, et al. Elevated maternal cortisol early in pregnancy predicts third trimester levels of placental corticotropin releasing hormone $(\mathrm{CRH})$ : priming the placental clock. Peptides. 2006;27(6):1457-1463.

36. Mesiano S. The endocrinology of human pregnancy and fetoplacental neuroendocrine development. In: Strauss J, Barbieri, R, eds. Yen and Jaffe's Reproductive Endocrinology. 7th ed. Philadelphia: Saunders Elsevier; 2009:243-271.

37. Smith R, Mesiano S, McGrath S. Hormone trajectories leading to human birth. Regul Pept. 2002;108(2-3):159-164.

38. Tyson EK, Smith R, Read M. Evidence that corticotropin-releasing hormone modulates myometrial contractility during human pregnancy. Endocrinology. 2009;150(12):5617-5625. 
39. McLean M, Bisits A, Davies J, Woods R, Lowry P, Smith R. A placental clock controlling the length of human pregnancy. Nat Med. 1995;1(5):460-463.

40. Hobel CJ, Dunkel-Schetter C, Roesch SC, Castro LC, Arora CP. Maternal plasma corticotropin-releasing hormone associated with stress at 20 weeks' gestation in pregnancies ending in preterm delivery. Am J Obstet Gynecol. 1999;180(1 Pt 3):S257-263.

41. Makrigiannakis A, Semmler M, Briese V, et al. Maternal serum corticotropin-releasing hormone and ACTH levels as predictive markers of premature labor. Int J Gynaecol Obstet Off Organ Int Fed Gynaecol Obstet. 2007;97(2):115-119.

42. Korebrits C, Ramirez MM, Watson L, Brinkman E, Bocking AD, Challis JR. Maternal corticotropin-releasing hormone is increased with impending preterm birth. J Clin Endocrinol Metab. 1998;83(5):1585-1591.

43. Hill JL, Campbell MK, Zou GY, et al. Prediction of preterm birth in symptomatic women using decision tree modeling for biomarkers. Am J Obstet Gynecol. 2008;198(4):468.e1-468.e9. 44. Polyakov A, Cohen S, Baum M, Trickey D, Jolley D, Wallace EM. Patterns of antenatal corticosteroid prescribing 1998-2004. Aust N Z J Obstet Gynaecol. 2007;47(1):42-45.

45. Swales D, Grande L, Wing D, Edelmann M, Glynn L, Sandman C, Smith R, Bowman M, Davis E. Data from: Can placental corticotropin releasing hormone inform timing of antenatal corticosteroid administration? Supplemental material. Figshare Digital Repository. Deposited 17 August 2018. http://doi.org/10.6084/m9.figshare.6934964.

46. American College of Obstetricians and Gynecologists. Management of preterm labor: Practice Bulletin No. 171. Obstet Gynecol. 2016;128(4):e155-164.

47. Behan DP, Khongsaly O, Liu XJ, Ling N, Goland R, Nasman B, Olsson T, Souza EB. Measurement of corticotropin-releasing factor (CRF), CRF-binding protein (CRF-BP), and CRF/CRF-BP complex in human plasma by two-site enzyme-linked immunoabsorbant assay. $J$ Clin Endocrinol Metab. 1996;81(7):2579-2586.

Figure 1 pCRH concentrations prior to betamethasone treatment predict time to birth. Figure Legend The Kaplan-Meier plot reports the probability of remaining pregnant, based on pCRH concentrations prior to betamethasone administration. Although all analyses were run continuously, for illustrative purposes we present the top and bottom quartiles of pCRH concentrations (top quartile in red, bottom quartile in blue). Women with the highest pCRH concentrations (top quartile, $82.60 \mathrm{pmol} / \mathrm{L}$ ) were more likely to deliver sooner, whereas women with the lowest pCRH concentrations (bottom quartile, $14.58 \mathrm{pmol} / \mathrm{L}$ ) were more likely to remain pregnant.

Figure 2 Receiver operating characteristic (ROC) curve (A) and sensitivity and specificity at each possible cutoff point (B) for placental corticotropin-releasing hormone (pCRH) at time of betamethasone treatment. Figure Legend Sensitivity indicates the probability that a case is correctly identified (true positive), and 1-specificity is the probability that a woman who will not deliver within 7 days is falsely identified (false positive).

Table 1. Maternal demographic and medical characteristics

\begin{tabular}{|l|l|}
\hline & Participants, $\mathbf{n}=\mathbf{1 2 1}$ \\
\hline Age at enrollment (yr) & $30.2(6.6)[18.6,46.9]$ \\
\hline Parity (\% Primiparous) & $29(24.0 \%)$ \\
\hline Ethnicity & \\
\hline
\end{tabular}




\begin{tabular}{|c|c|}
\hline Non-Latina White & $27(22.3 \%)$ \\
\hline Latina & $77(63.6 \%)$ \\
\hline Other & $17(14.0 \%)$ \\
\hline Gestational Age at Assessment (wks) & $28.4(3.2)[23.0,33.9]$ \\
\hline Body Mass Index $\left(\mathrm{kg} / \mathrm{m}^{2}\right)^{a}$ & $31.3(0.6)[19.1,46.5]$ \\
\hline Married or cohabitating & $98(81.0 \%)$ \\
\hline Years of education & $13.37(2.89)[2.00,21.00]$ \\
\hline \multicolumn{2}{|l|}{ Income to Needs Ratio ${ }^{b}$} \\
\hline$<1$ & $36(32.4 \%)$ \\
\hline $1-2$ & $33(29.7 \%)$ \\
\hline $2-4$ & $17(15.3 \%)$ \\
\hline$>4$ & $25(22.5 \%)$ \\
\hline \multicolumn{2}{|l|}{ Indication for Betamethasone Treatment } \\
\hline Placental abnormalities & $27(22.3 \%)$ \\
\hline Inflammation & $77(63.6 \%)$ \\
\hline Utero-placental insufficiency & $17(14.0 \%)$ \\
\hline \multicolumn{2}{|l|}{ Obstetric risk factors } \\
\hline Preeclampsia & $11(9.1 \%)$ \\
\hline HELLP & $1(0.8 \%)$ \\
\hline Preterm premature rupture of membranes (PPROM) & $27(22.3 \%)$ \\
\hline Gestational diabetes & $14(11.6 \%)$ \\
\hline Diabetes & $6(5.0 \%)$ \\
\hline pCRH (pmol/L) & $69.9(102.0)[0.25,600.0]$ \\
\hline
\end{tabular}

Notes:

Mean (SD) [Range] are presented for continuous variables, and $\mathrm{N}(\%)$ are presented for categorical variables.

${ }^{a} \mathrm{BMI}$ is missing 4 participants.

${ }^{\mathrm{b}}$ INR is missing 10 participants.

Table 2. Neonatal medical characteristics

\begin{tabular}{|l|l|}
\hline & Participants, $\mathbf{n}=\mathbf{1 2 1}$ \\
\hline Gestational Age at Birth (weeks) & $34.3(4.7)[24.3,40.7]$ \\
\hline Preterm (<37 weeks) & $69(57.0)$ \\
\hline Birth weight (g) & $2336.20(1008.33)[570,4480]$ \\
\hline Apgar score at 5 $\min ^{\text {a }}$ & $8.00[1,10]$ \\
\hline Sex (\% Male) & $69(57.0 \%)$ \\
\hline
\end{tabular}

Notes: Mean (SD) [Range] are presented for continuous variables, and $\mathrm{N}(\%)$ are presented for categorical variables.

${ }^{\mathrm{a}}$ Apgar score is presented as Median [Range].

Abbreviations: ACOG, American College of Obstetricians and Gynecologists; AUC, area under the curve; BMI, body mass index; GAB, gestational age at birth; pCRH, placental corticotropin-releasing hormone; RIA, radioimmunoassay; ROC, receiver operating characteristics. 


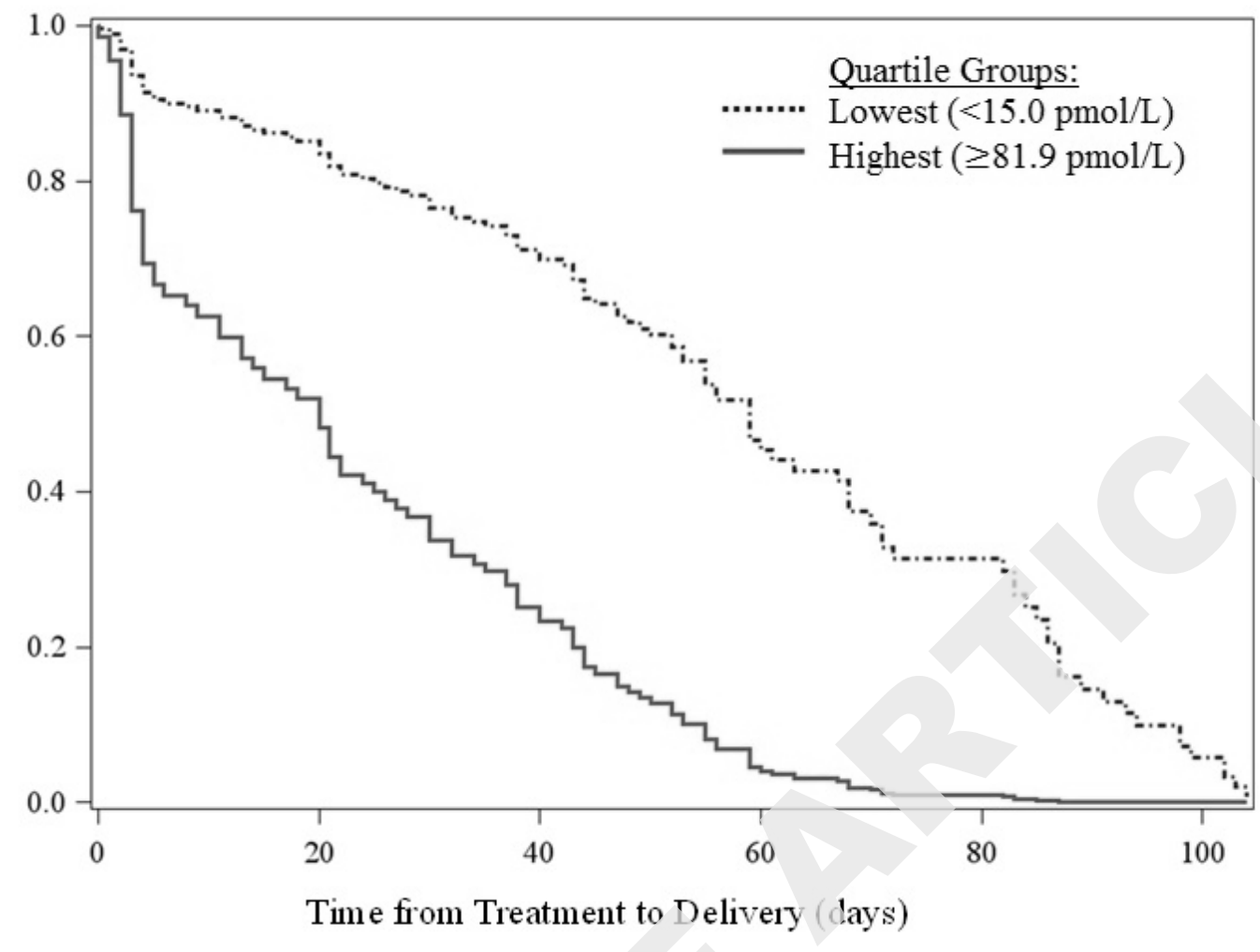



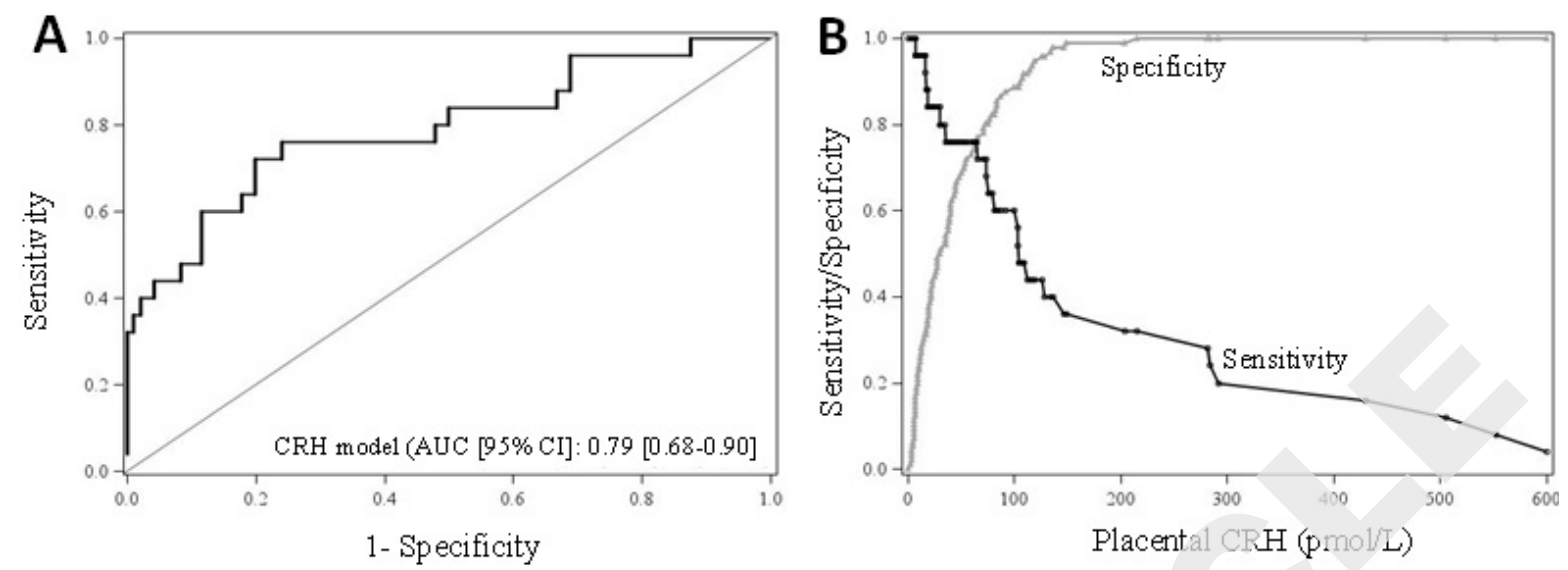